\title{
Innovations in Flexible Endoscopic Evaluation of Swallowing and Laryngeal Sensation Testing
}

Shamik Paresh Mehta

\section{ABSTRACT}

The larynx plays a crucial part in swallowing to prevent aspiration. The present available clinical tools to assess this function of larynx are 'flexible endoscopic evaluation of swallowing' (FEES) and 'laryngeal sensory tests'. The author has made certain changes to make these tests equally effective and economical.

Keywords: Aspiration, FEES, Laryngeal sensations, Penetration, Swallowing.

How to cite this article: Mehta SP. Innovations in Flexible Endoscopic Evaluation of Swallowing and Laryngeal Sensation Testing. Int J Phonosurg Laryngol 2015;5(1):17-19

Source of support: Nil

Conflict of interest: None

\section{INTRODUCTION}

The most primitive function of the larynx is valvular. It senses the bolus via the pharyngeal branches of the vagus and the superior laryngeal nerve and closes itself by laryngeal adductor reflex, and thus it protects the lower airway during swallowing.

The pharyngeal phase of swallowing starts with the closure of the vocal folds, false cords and laryngeal inlet by epiglottis. With the help of the strap muscles, the larynx is brought forward and upwards opening the upper esophageal sphincter. Simultaneously, the base tongue acts as a piston and pushes the bolus contents into the upper esophagus.

This co-ordinated process becomes abnormal in a number of physical conditions. Therefore, the swallowing assessment becomes important to prevent aspiration.

Gag and cough reflexes were the only swallowing assessment tools used before Susan Langmore introduced flexible endoscopic evaluation of swallowing (FEES) and Jonathan Aviv came with the laryngeal sensory tests. ${ }^{1,2}$

Consultant

Department of ENT, Sushrusha Hospital, Ahmedabad, Gujarat India

Corresponding Author: Shamik Paresh Mehta, Consultant Department of ENT, Sushrusha Hospital, Navrangpura Ahmedabad, Gujarat, India, Phone: +91-9879090765, e-mail: mehta.shamik@gmail.com

\section{The Technique}

Traditionally, the laryngeal sensory tests are done first followed by the FEES.

The flexible video laryngoscope is used to observe and document the findings.

\section{Laryngeal Sensory Tests}

The tip of the laryngoscope is kept $5 \mathrm{~mm}$ away from the target tissue. A separate channel in the scope or a channeled sheath is used, the other end of which is attached to an equipment called 'air pulse generator' to deliver the pulsed air to stimulate the larynx. As the laryngeal mucosa is stimulated by the gusp of air, the laryngeal adductor reflex will be observed. There is a facility to regulate the pressure at which the air is delivered, and thus one can quantify the loss of sensations as mild, moderate or severe. ${ }^{3}$

Some institutes at which this facility is not available, use so called 'tapping the tissue with the scope', and observe/record the findings.

\section{Our Innovations}

We use chip-on-tip video rhinolaryngoscope to observe and document the findings. We use channeled sheath (Fig. 1).

Instead of air pulse generator, we use the compressorbased nebulizer to stimulate the laryngeal mucosa. We keep the tip of the scope (where one end of the channeled sheath lies) $5 \mathrm{~mm}$ away from the target tissue, stimulate the mucosa with the nebulizer air current and see/record the laryngeal adductor reflex (Fig. 2).

The advantage is obviously the cost and availability. Whereas the air pulse generator is very costly and not easily available, the commonly used nebulizer is readily available and at a minimal cost.

The disadvantage is that it will not give the quantitative estimation of the laryngeal sensations and will give information about the presence and absence of the laryngeal adductor reflex only.

This innovation is much more specific and objective as compare to the 'tapping the tissue with scope' technique. ${ }^{4}$

\section{Flexible Endoscopic Evaluation of Swallowing}

The tip of the video laryngoscope is kept at the level just above the base of tongue and the patient is asked to 


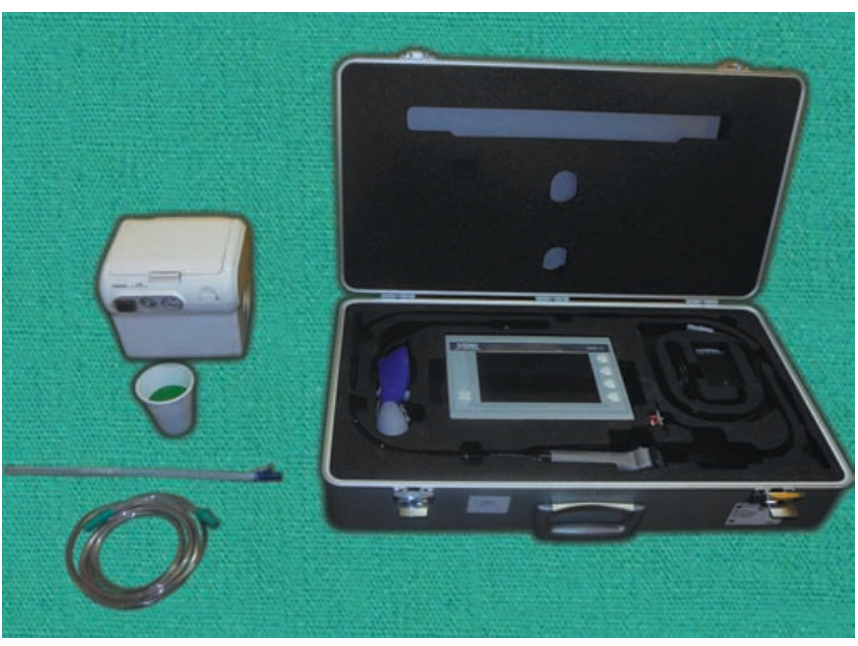

Fig. 1: The equipment

swallow the food of different consistency, i.e. liquid, semisolids and solids colored with appropriate food colors, and the videos are documented. 5,6

\section{Our Innovations}

We use only colored normal saline as a swallowing material. Normal saline is the thinnest material, most likely to get aspirated, but least likely to cause any damage to the lungs. Therefore, this makes the test safer and more sensitive.

We keep the tip of the scope just above the base of tongue and below the uvula and ask the patient to swallow the green colored saline.

We assess the post-swallow residue in vallecula and pyriform fossa; penetration; aspiration and spillage (Figs 3 to 5). The penetration aspiration score gives better idea about further management. ${ }^{7}$

\section{MATERIALS AND METHODS}

A prospective study was carried out under the supervision of an independent ethics committee of Ahmedabad.

Twenty-eight patients were included in the study who were having recurrent or threatened aspiration between July 2013 and June 2014.

Seven patients had undergone extensive head-neck surgeries with or without radiotherapy, six patients had Parkinsonism, three patients had progressive supranuclear palsy (PSP), four had cerebrovascular stroke, two patients had skull base tumors, two patients with metabolic encephalopathy and four patients did not have any identifiable pathology, but had history of single or more attacks of aspiration pneumonitis.

All patients underwent swallowing assessment in form of flexible endoscopic evaluation of swallowing and laryngeal sensory tests as per our innovative techniques as described earlier.

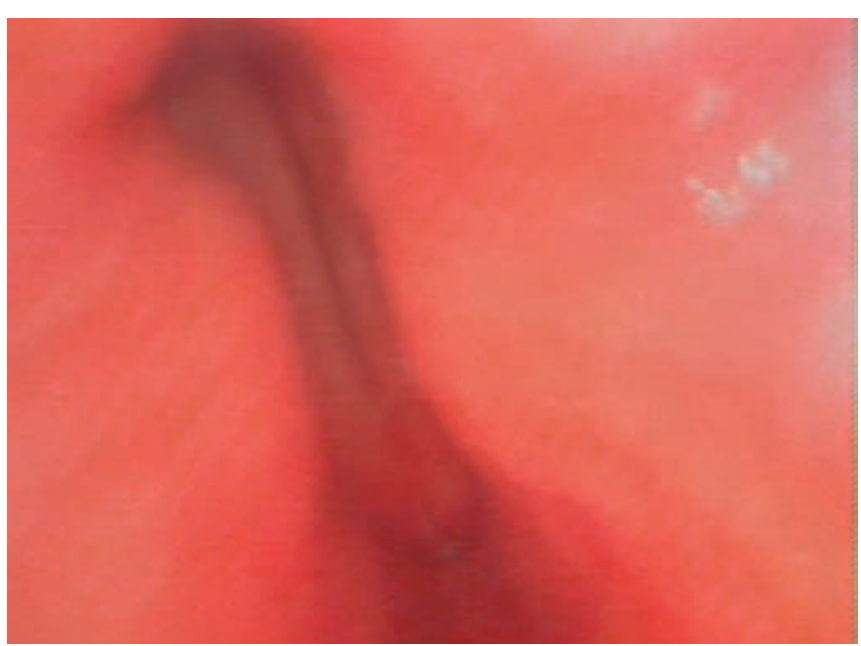

Fig. 2: The laryngeal adductor reflex

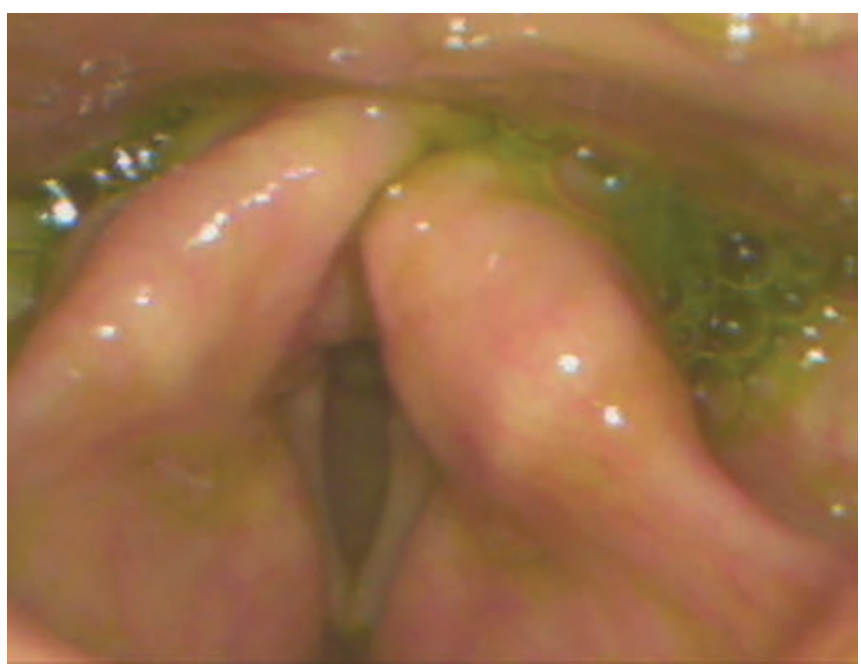

Fig. 3: Fees in LVC palsy

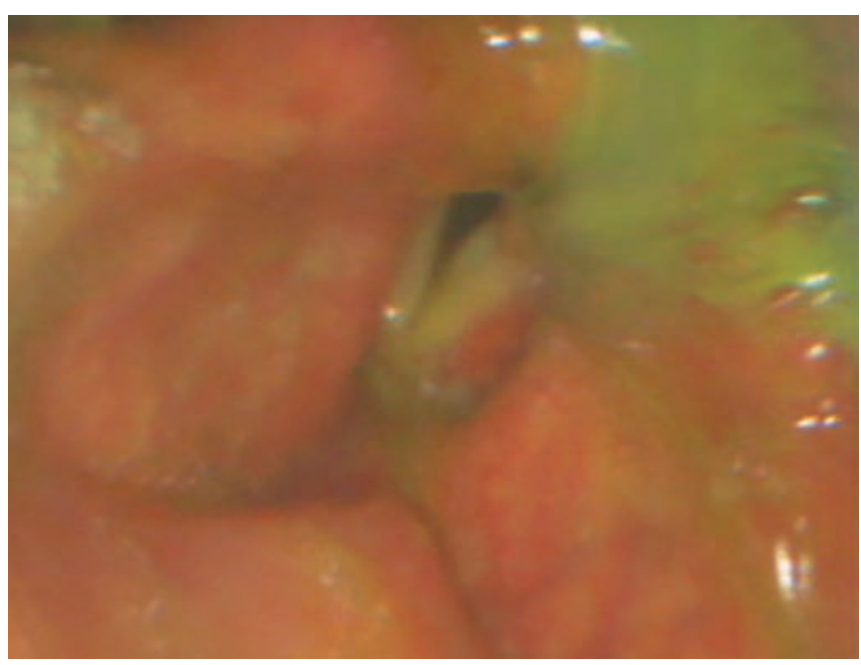

Fig. 4: Penetration

Depending upon the swallowing assessment, the patients were advised as follows:

- Swallowing positions/manoeuvres/exercises in 20 patients.

- Temporary nasogastric tube in 10 patients. 


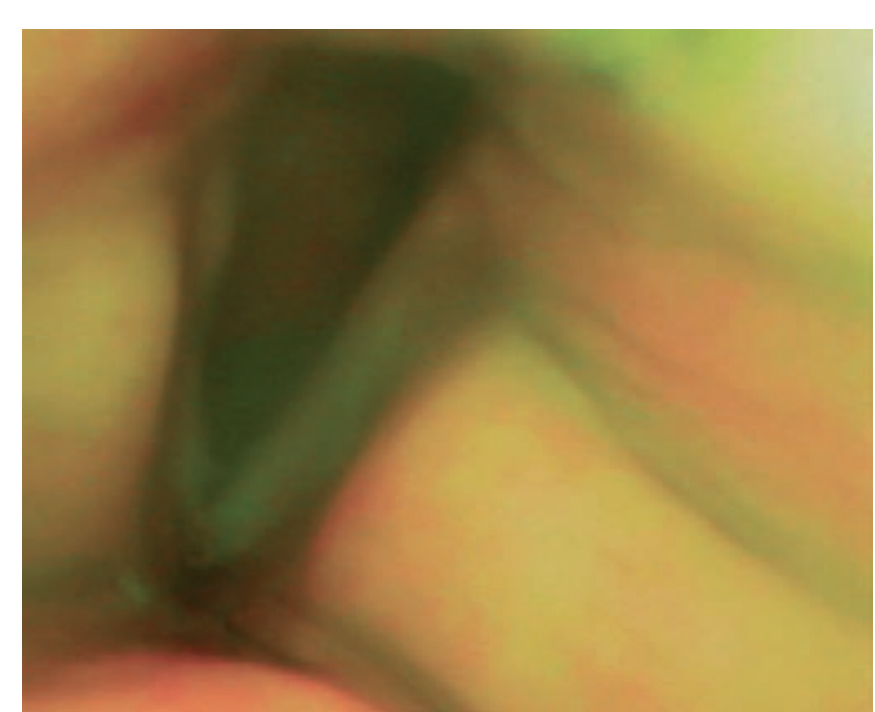

Fig. 5: Aspiration

- Gastrostomy in 10 patients.

- Tracheostomy in two patients.

All of these patients were followed for 6 months to 1 year.

\section{RESULTS}

One patient died due to extensive skull base malignancy. None of the patients showed aspiration pneumonitis after the assessment and therapy.

In five patients, we were able to take them off the nasogastric tube immediately after the swallowing assessment.

Two patients required follow-up swallow assessment which showed improvement.

At present, 12 patients are swallowing normally. Five patients still experience occasional cough while swallowing liquids.

\section{CONCLUSION}

The FEES and laryngeal sensory tests are, by far, the most sensitive tests for proper transit of bolus from pharynx to esophagus without aspiration. ${ }^{6}$

The use of nebulizer for laryngeal sensory stimulus is more specific as compared to the tapping of tissue with scope or the double scope method. It is much more economical and easily available as compared to the air pulse generator. However, it does not give any quantitative information about the sensory loss of laryngeal mucosa.

The use of normal saline makes the test more sensitive as saline is the thinnest material to get aspirated and safest material with regard to lung damage.

\section{REFERENCES}

1. Langmore S, Schatz K, Oslen N. Fiberoptic endoscopic examination of swallowing safety: a new procedure. Dysphagia 1988;2(4):216-219.

2. Aviv JE, et al. Flexible endoscopic evaluation of swallowing with sensory tests: a new bedside endoscopic test of the motor and sensory components of swallowing. Ann Otol Rhinol Laryngol 1998 May;107(5 pt 1):378-387.

3. Shaker R, et al (eds). Manual of diagnostic and Therapeutic Techniques for Disorders of Deglutition. New York: Springer Sciences + Business Media 2013. DOI 10.1007/978-1-46143779-6_6.

4. Bastian RW. Videoendoscopic evaluation of patients with dysphagia: an adjunct to the modified barium swallow. Otolaryngol Head Neck Surg 1991;104(3):339-350.

5. Shaker R, et al (eds). Manual of Diagnostic and Therapeutic Techniques for Disorders of Deglutition. Springer Sciences + Business Media:New York 2013 DOI 10.1007/978-1-4614-3779-6_5.

6. Murray T, Carrau RL. Clinical management of swallowing disorders. 3rd ed. Evaluation of Dysphagia 2012;13:978-159756-425-0.

7. Rosenbek JC, Robbins J, Roeker EB, Coyle JL, Woods JL. A penetration and aspiration scale. Dysphagia 1996;11(2):93-98. 